This is not the version of record. The full version of Chapman, Daren and Wang, Stephen Jia (2020) Individual 'trace' in knowledge space : a novel design approach for human-systems interaction. in Ahram, Tareq, Taiar, Redha, Gremeaux-Bader, Vincent and Aminian, Kamiar (eds.) (2020) Human Interaction, Emerging Technologies and Future Applications II. Advance in Intelligent Systems and Computing (1152) Cham : Switzerland, pp. 219-224. ISBN: 9783030442668 ISSN (print) 2194-5357 ISSN (electronic) 2194-5365. can be found at https://doi.org/10.1007/978-3-030-44267-5_33 


\title{
Individual 'Trace' in Knowledge Space: A Novel Design Approach for Human-systems Interaction
}

\author{
Damian Chapman ${ }^{1}$, Stephen Jia Wang² \\ ${ }^{1}$ The Design School, KSA, Kingston University, KT1 2QJ, UK \\ damian.chapman@kingston.ac.uk \\ 2School of Design, The Hong Kong Polytechnic University, \\ stephen.j.wang@polyu.edu.hk
}

\begin{abstract}
Data mining design is an approach through which system operational improvements in the search and retrieval of data activity can be augmented. This study explores optimisation processes, including data harvest, analytics and visualisation plus covers a wide range of efforts, including identifying the growing need of 'making-sense' of data which requires contextual understanding. In both cyberspace and physical world experiences the exploring of challenges and linkages between the cyber-physical knowledge spaces in data are emerging with excessive amounts of raw data.

Possibilities to improve User-interface-design through better visualisation infographics in this study propose a novel mapping approach called 'Trace' in the Knowledge Space enabling design opportunities that help articulate unique human-system interaction, which provide potential in re-imagining and re-structuring uses of interaction and userexperience. These experienced through the design, use and context of languages enabling the building of new interactive apparatus, algorithms and dynamics in collective intelligence.
\end{abstract}

Keywords: Human-systems Interaction · Knowledge Space · Individual Trace · Second Order Cybernetics · User Interaction Design

\section{Introduction}

Operational improvements in the search and retrieval of data enabling greater breath of understanding how to utilise and augment human and machine productivities are central to developing cultures of efficiency. New software models refined through precision algorithmic Machine learning are leading optimisation and approaches for heightened human-machine hybridity, requiring greater capability in understanding data analytics to achieve better intelligence. We believe this approach can be further improved through increasing Artificial Intelligence capabilities in learning and enabling data predictions and re-structuring the relationships between machine and human interaction. Creativity and opportunity are 
embedded and await articulation through content from texts, imagery, networks of libraries and archives and the complex mass of data. The Individual Trace in the Knowledge Space approach proposed in this paper could help in this sense to augment insights understanding therefore to enhance human-system interactions.

Speech input in interface design drives embodied interaction enhancements in Systems design, with an added component to the previously more dominant visual graphic interfaces connecting and engaging users monitoring, analysing and visualising data. However, there is an emerging trend in 'always-on' connectivity available, such as in-ear systems, speech and the more haptic, gestural or optically driven technologies. This new expanded interface optionality utilising a broader range of sensory input mechanisms creates a more embodied user as co-bot partnership. Working in highly technologically advanced networks, design is evolving into a more progressive human-plus-machine hybridity [1].

Heightened integration of human interaction by emerging technologies has led to the design of cloud-based and web 4.0 applications. Integration incorporates a range of gestural and voice interaction thereby creating greater scope for engagement in and through user interface innovations such as application programming interfaces. The developments for improving human-system communication and interaction continue further developments in designing better design processes and greatly enhancing co-design methods to further support heightened user-experience, interaction capacity and productivity efficiencies.

In order to pivot the context of the individual's embodiment within the footprint or trace of emerging and adapting libraries, it is useful to revisit the interaction and meshwork of connections. In this paper, there are two inter-related sections:

Firstly, introduce the concept of Enhancement of Human-Systems-Interaction (HSI) through literature review in the fields of Hyper-linked search and retrieval, Second Order Cybernetics and Spatialised Cognition understood through a Constructionist approach.

Secondly, propose a novel method called 'Individual Trace in the Knowledge Space' (ITKS). ITKS is a research framework which aims to create a new approach in methodology to further understand the potential in efficiencies to sort, filter and re-structure data over-load by mapping data communication trajectories and interactive-relationships transforming raw data through analytics, modelling, simulation and optimization for enhanced User Interface design.

\section{Enhancement of Human-Systems-Interaction (HSI)}

Enhancements in human-systems design for interaction are understood through bespoke interactive coded language. The variety of which are represented from raw through to highly dedicated algorithms as building blocks in input data. These differing inputs of coded 'languages' are used to drive and augment access to the loT as well as indigenous 
knowledge spaces like libraries, industry operations systems and bespoke digital archives. Jon Kleinberg's research from the perspective of HITS: Hyperlink-Induced Topic Search is known also as the Hubs and Authorities algorithm [2]. HITS algorithm is interesting in its application from Hyperlink-Induced linked search and retrieval being one of the key algorithmic influences for the success of Google's evolving search engine. Kleinberg's research on 'Authoritive Sources in a Hyperlinked Environment' [2] presents ideas for making sense of how an emerging network of hyper-linked hubs can point at or 'join' authoritative sets of collective intelligence in Web-based archives. This approach contextualizes content in an agile network of Hubs that point to continually updating Authoritive knowledge sources.

To build on Kleinberg's ground-breaking studies Gary Flake's research from the context of trace within data search legacies focuses on Communities of similarity in search \& retrieval data characteristics [3]. The 'Community of similarity' algorithm [4] that was developed being instrumental to the construction of the innovative 'browser' interface Flake named Pivot whilst leading the Live labs research studio at Microsoft [4]. The 'Community of similarity' algorithm [3] built from a parallel mindset to that of Page-rank in open-source browsers like Google, Firefox and Opera amongst others, with search interaction being designed through adaptive filtration of data subsets in search and retrieval interaction creating a diverse search engine functionality. The idea underpinning Communities of similarity in hyperlinked pages/phrases or strings of data is a useful comparator of relationships to the 'HITS' Hyperlink-Induced Topic Search [2] that ranks use-case searches and therefore constructing an evolving taxonomy related to network content through both the context of text, image, dates and publication origins.

The Pivot browsing utility interfacing internet 'web' connectivity through visualisation geometry creating 'Collections'[4]. Collections being grids of grouped information presented through image matrices in context to data search streams in a unique adaptive browser experience in a live infographic. Through zooming into the dense visual patterns of data imagery, the user has access to dynamic interaction rather than a linear search and retrieval presentation for a browser search tool. This has the capacity to transform data in context to both glanceable infographics and schema structures mining the workflow into 'Communities of pages' in a recursive exploration of content [4].

Interaction in archives through recursive activities supporting communities of content and ranking in emergent search hierarchies of data evolves the capacity of machine learning, augmenting $\mathrm{Al}$ in smarter algorithms to search browsers. This likewise informs the methods for designing improved efficiencies in digital interaction. To enable greater variety in interaction 'actors' and can be understood as an approach that provides insights for design automation and new discoveries for innovating learning. In other words, the increased knowledge from a broader approach to collaborative machine and human collective intelligence applied to observing, capturing, and adapting communities of data as well as 
fulfilling search and retrieval interaction objectives can achieve a greater organisational and productive goal set [7]. This interaction achieved through a navigational cycle steering designed interactive 'conversations' [5] being organised between the stakeholders involved in objectives and aims of industry. The interaction step-by-step method being compared to and named 'conversations'. The design and evaluation objectives fulfilling human interaction through setting goals via a circular nature of Systems-design [5]. Likewise designing understanding to improve the potential of 'wants' from what is presented and adapting this data in a First order approach via Search engine feedback. Second Order systems design adapts the user experience by observing and participating in the activity of search. this is likened to the helmsman of a ship, steering and adapting to weather conditions to navigate to a chosen destination [5]. 'Conversation theory' was developed by Gordon Pask originally with Paul Pangaro further developing this subject through research both celebrating and curating Pask's work. However also building on Conversation theory applied to Second Order Cybernetics in a number of live projects that explore designing ethics in interaction and coevolutionary design in organizations and society [5]. Pangaro's research and work exploring the design of interaction through mapping out focus aims within conversations enables resource needs to fulfil a process whereby interaction with actors is designed to facilitate knowledge that contributes to resolving transactions and fulfil set goals [5].

\section{$3 \quad$ Individual Trace in the Knowledge Space (ITKS)}

From 2015 Web3.0 functionality has grown from an interactive and social network approach that facilitates collaborative search, retrieval and content input to the current Web4.0 creating shifts in the dynamic stream of communication data flow from both human and non-human input [1]. From this input ITKS as a research framework can further interrogate the relationships between the individual trace and group trace data applied to local indigenous and larger collective knowledge spaces. From ITKS is the potential for efficiencies to sort, filter and re-structure data over-load by mapping the data communication trajectory and interactive-relationships within systems organizational agile shapes and structures [6]. From this divergence in raw data analytics, new understanding in observing systems, simulation through 'Digital twin' design and optimization for vision streaming can enhance Human interaction and emerging technologies [6].

Digital interaction between individual actors is able to transcend barriers of human to human interaction through developing sophistication in network interactivity augmenting capacity from Al driven efficiencies. To build on Daugherty and Wilson's 'Missing middle' approach [1], industry technology needs to address how human interaction can train Al through machine learning to compliment the leadership and creative empathy that human operators input. Machines have the potential to deliver speed, analytics, super strength and accuracy in machine and Al led transactions, adaptations and productivity. However human and machine 
hybridity can amplify and interact to augment Human-Interaction with a far greater and more embodied symbiosis in the relationships between human and machine [1].

Individual Trace in the Knowledge Space as a new approach can augment insights from the 'Missing middle' [1] to greatly enhance communication through Al. From an industrial perspective, focused on human interaction for the iterative improvement through design of systems augmenting production capacities and safety in the Oil and Gas production operations. Now timely and needing research to resolve due to growing data overload and the need for insights to improve search, retrieval optimization from increasingly diverse groups of stakeholders. The research also focuses on the deeper understanding of production efficiencies in emergent live stream data-flow to build 2 nd order functionality in operations presenting a crucial data visualisation method in intelligent manufacturing [6].

Building on how data trails and trace enabled from human and non-human interaction, be it industry focused productivity or sensor driven Al through Machine learning between production and consumption capacities. From the perspective of content consumption in the larger internet Knowledge Space mankind struggles to cope with the mass of data overload and data-exhaust. Data-lake repositories and archives are enhancing an emergence in data work-flow in unprecedented quantities from the ever-increasing streaming of data input. However there is a crucial need for customizable algorithms that can analyse, model, simulate and optimise data to develop agile schema structures and models for transforming raw data into productive insights and knowledge. Clarity from individual data streams, triggers and alarm calibration with capacity improvements are the goals for new insights. Using a range of transactions and streaming adaptations shifting finite responses to live monitored flow statistics. The comparisons to Social Media, and classification in interaction user-experience are gaining phenomenal technological shifts to now re-imagine and reshape communication tool-kits [1, 7]. The user experience in interaction between human to human in online environments and the potential of increased capacity working with the dramatic shifts in machine learning for human and machine hybridity through collective intelligence central to operational productivity opportunities. The issues in data overload requiring new thinking and a gap in knowledge for transforming data overload into Individual Trace in the Knowledge Space.

When exploring Second Order functions in human interaction and the design of emerging technologies Cybernetics and its circular observational loop of design improvability is a central approach. Designing the design through an approach both from the perspective of an iterative methodology and a rich innovative philosophy behind Web4.0. The circular approach of Second Order Cybernetics, observing the observing in the design feedback cycle being built into individual trace in operations, and in their system meshworks with Individual trace in indigenous Knowledge Spaces becoming central for the visualisation in feedback loops as forms of Circularity [5]. The aim being to learn how to make better 
decisions in the use of data-flow through an agile approach that enables interaction, repeated cycles of learning designed in to the methodology. From this method called the Iterative Design Development Cycle [6] improvements can be designed through phased aims and productivity efficiencies. Intuitive design enhancements for technology are better driven by second order feedback loops in systems design to build in more flexibility for learning and augmenting streaming improvements.

\section{Discussion: Iterative Design Development cycle (IDDC)}

The development of the Iterative Design Development cycle [6] has derived from a mixed methods approach where the research is constructed from a 'thinking through making' philosophy, prototyping and gaining user-feedback to innovate insights. The design method developed from Eric Ries's influential constructionist approach supporting an iterative learning cycle originating in digital based Business StartUp's [8]. Ries's methodology involves a Minimum Viable Concept (MVP) and Minimum Viable Product (MVP) being exposed to an early-adopter market to engage user feedback [8]. The Iterative Design Development Cycle [6] develops from a Co-design, action research and literature insights through a feasibility iteration with a Make cycle to Co-design and understand user needs and wants. Then iterated in a Usability Measuring through user-testing, developmental prototyping and Review documentation. The practice of making prototypes rather than a priori as an approach from a theoretical approach [6]. The 'Build / Measure / Learn' iterative methodology [8] designed and developed creating a methods design toolkit involving feasibility, usability and improvability cycles in three phases: Make / Measure / Insights [6]. These interactive circular approaches evolving development through insights from live Usecase insights to inform a research development cycle. Data flow used constructively for reimagining the design connecting data in dynamic systems. This new approach utilises Machine learning to process data-flow and identify assets through $\mathrm{Al}$, then mapping the Trace within live data model streams.

\section{Conclusion}

The research in Individual Trace in the Knowledge Space originated from working in industry as a design consultant and experiencing a gap in knowledge around working with data insights and the global problem of data-overload. The trace of workflow from an industrial and experiential cultural trace in domestic perspectives as germination points for new visualisation tools, improved UiD and UX is needed plus a framework to explore individual trace is key in the growing Knowledge Space. Clearly there is a timely need for new knowledge to better understand, design and implement improvements to the data trails and trace that transform the aggregate efficiencies in both user experience for search and 
retrieval, supply and delivery and through interaction with data for greater user potential. It is possible that through improvements to visualisation methods in data harvest, intelligence and productivity efficiencies using a new ITKS framework that individual trace of data trails can be utilized in the emergence of new approaches in what is referred to as the 4th Industrial Revolution: Cyber-physical systems constructing the Knowledge Space through data-trace and the intelligence being created in the mushrooming 'always on' inter-connected Knowledge Space of 'the Cloud', 'Data-lakes' and Internet of Things.

\section{References}

1. Daugherty, P., Wilson, J.: Human + Machine: Reimagining Work in the Age of Al. Harvard Business Review Press (2018)

2. Kleinberg, J.: Authoritative Sources in a Hyperlinked Environment, NY (1997)

3. Flake, G.: The Computational Beauty of Nature: Computer Explorations of Fractals, Chaos, Complex Systems, and Adaptation. MIT Press, Cambridge (2000). New Ed.

4. Flake, G.: Gary Flake on Overture, Yahoo and the History of Search (2017). https://www. youtube.com/watch? $v=g Q d 7 V w N a 0 j E \& t=1488 s$

5. Pangaro, P.: The future of Cybernetics. Lecture Nano World's Fair, San Francisco (2018)

6. Chapman, D., Wang, S.J.: Trace: A Crucial Data Visualisation Method in Intelligent Manufacturing (2019)

7. Malone, T.: Superminds: The Surprising Power of People and Computers Thinking Together. Oneworld publications, London (2018)

8. Ries, E.: The Lean Startup. Penguin Books Ltd., London (2011) 